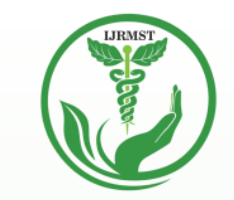

INTERNATIONAL JOURNAL OF RESEARCH IN MEDICAL SCIENCES \& TECHNOLOGY

e-ISSN:2455-5134; p-ISSN: 2455-9059

\title{
HAIR LOSS: CAUSES AND PATHOLOGY
}

\author{
Mohammed D. Mahmood \\ Department of Pharmacology and Toxicology, College of Pharmacy \\ University of Mosul, Mosul, Iraq
}

Paper Received: 30 ${ }^{\text {th }}$ March, 2021; Paper Accepted: 09 ${ }^{\text {th }}$ May, 2021;

Paper Published: 29 ${ }^{\text {th }}$ June, 2021

DOI: http://doi.org/10.37648/ijrmst.vl li01.016

How to cite the article:

Mohammed D. Mahmood, Hair Loss:

Causes and Pathology, IJRMST,

January-June 2021, Vol 11, 167-176, DOI:

http://doi.org/10.37648/ijrmst.v11i01.016

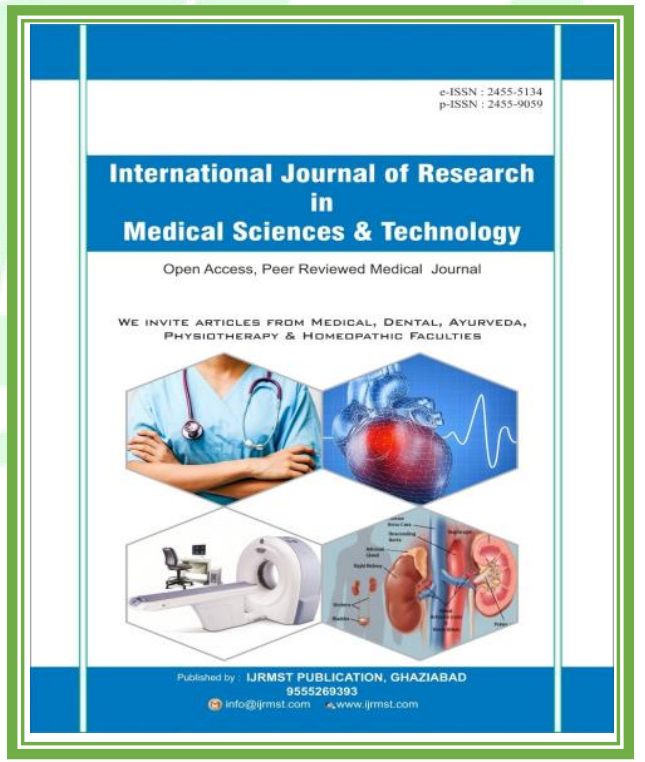




\section{ABSTRACT}

There are many diseases in which one of the main symptoms is hair loss. Hair loss in medical terms is called "Alopecia". Many reasons can cause alopecia. Hair loss can be due to nutritional factors, genetic problems, metals toxicity, and body inflammation. Hormonal changes in the body can also lead to a loss of hair. Physiology and stages of reproduction of hair in humans are also discussed. There are three stages in the process of the hair reproduction cycle. Many factors can be responsible for the disruption of hair, and stress is one of them. A hair pulling test should be done to check the quality of hair. Doctors prescribe the medication and treatment according to the cause of the hair loss. If the hair loss is due to nutritional changes, proper diet and supplements should be taken to reduce the hair loss. A clinical evaluation of a patient with hair loss is important to conclude. Doctors should keep themselves refreshed with the new advances in treatment. Age factor is one of the primary reasons for hair color changes and hair loss. Trichotillomania and traction alopecia are the two types of self-inflicted hair loss that involve pulling the hair and heat, worsening hair condition using hair straighteners, respectively.

Keywords: hair loss, alopecia, etiology, physiology.

\section{INTRODUCTION}

Many diseases are associated with hair loss. Alopecia is the term used for hair loss conditions. Hair loss can occur in both men and women. Age usually does not matter in the condition. Hair loss has a substantial impact on social and psychological well-being. As there are many causes for alopecia, a complete medical history and the complete study on hair loss and a few basic tests can quickly control the chances of causing hair loss. One of the most common types of hair loss condition is androgenetic alopecia. It has a distinct pattern of hair loss in men and women. Topical minoxidil has been licensed by the US FDA to treat both men and women, including finasteride for men. The loss of "handfuls" of hair is a symptom of telogen effluvium, which responds to emotional or physical stress. On examination, alopecia areata has distinct characteristics that aid in diagnosis. The hair in the scalp usually grows about $0.35 \mathrm{~mm}$ (6 inches per year) per day. About 100 hairs per day are shed by the scalp and even more when shampooed. Because each hair follicle passes across the triple-staged growth independently, observing the hair loss process is not even noticeable $(1,2)$.

It is possible to treat many of the causes of hair loss in children. As a result, early detection is critical for accurate handling 


\section{VOLUME: 1 1, JANUARY-JUNE 2021}

to prevent further hair loss and

complications such as alopecia. In dermatological practice, hair loss in children is a common issue and for dermatologists is regarded as a challenge.

The roots of hair loss in younger humans have been categorized based on the alopecia pattern, which includes focal patches on the scalp and scars on the skin. Different conditions can be responsible for causing hair loss in people. Hair loss can be one of the major signs in different medical conditions like genetic, autoimmune, and pathogenic infections. Hair loss is caused when the immune system targets the hair follicles. Some external factors are also included in causing hair loss in people (3). Alopecia can be induced by insufficient intake of nutrients, genetic problems, weakened immune system, or side effects of some drugs. The most common class of drugs that induces alopecia is psychotropic drugs. Research has been made to understand the mechanism of induction of alopecia due to drugs. However, the reason behind this is still unknown. Selective reuptake serotonin inhibitors are the most common psychotropic drugs that fall under the anti-depressants responsible for alopecia. A side effect of the drugs is bound to hair loss and is also responsible for changing the color, structure, and texture of the hair $(1,2,4)$.

The bodily exam must be taken of the hair and scalp. However, interest must accept bodily symptoms and symptoms of any comorbid disorder indicated using the evaluation of systems. If most effective the hair in the scalp is involved, the health practitioner must search for the usual male or lady sample to decide the presence of the type of alopecia. Whole hair loss is dependent on the type of alopecia. Dry, damaged hair indicates trichorrhexis nodosa, while scaling, pustules, crusts, erosions, erythema, and neighborhood adenopathy endorse infection. Hair loss conditions can be checked by performing a "pull check" test in both men and women. About 40 to 60 hairs at their base are being taken by the examiner using his thumb, index, and center palms and apply mild traction way far from the hair of the scalp. A high-quality result is when more than $10 \%$ of hairs (4 to six) are being pulled from the scalp; this means energetic hair losing and indicates a telogen effluvium, anagen effluvium, or another kind of alopecia. However, a poor check result now no longer excludes one's conditions. The following test does not provide accurate results and is not considered standard because the pulling pressure isn't dispensed monotonously and because it is 


\section{VOLUME: 1 1, JANUARY-JUNE 2021}

miles tough to approximate the range of hair taken, thereby interpretations being made in which there might be some false results $(3,4,5)$. Having hair loss is a typical condition. It very well may be brought about by an assortment of problems that can be inborn or gained. The capacities of getting doctors to comprehend the etiological variables and the distinctions between these issues assist them with arriving at the right analysis and treatment. A careful clinical evaluation of a patient with hair fall is important to conclude. Doctors should keep themselves refreshed with the new treatment advances $(1,2,6)$.

\section{HAIR GROWTH: NORMAL PHYSIOLOGY}

Hair is a part of the integumentary system that extends into the dermal layer, which is contained in a hair follicle. Hair is a fundamental distinguishing feature of mammals as distinct species. It is a prized and visible indicator of health, youth, and even social class. It has a sensory role, defends against cold and UV radiation, and when its growth or structure is disrupted, it can have a substantial psychological impact. The variation in length, color, diameter, and cross-sectional form of each hair provides the distinctive profiles seen between ethnic groups and individuals on a microscopic level (7). There are two main different structures present in the hair. One of them is the follicle in the skin and the other is the hair shaft which clearly can be seen on the body surface. The hair shaft is made up of cortex and cuticle cells. In the central region, a medulla is present in some cases. The medulla is the hair's core portion. The follicle is the hair's primary growing structure. The following histological structures can be seen on the follicle's outermost surface:

- The outer root sheath (ORS), identified, is number one. It comprises keratinocytes and serves as multipotent stem cells. These cells are the keratinocytes and melanocyte stem cells.

- The inner root sheath is the other main part which is comprised of cuticle layers. The hair shaft cuticle is being attached to the inner root sheath cuticle layer. The follicle to the hair shaft Keratins is produced by IRS cells and trichohyalin, which acts as an intracellular cement giving the IRS the strength it needs to hold and sculpt the hair shaft as it grows well as leads its journey to the upside. The inner root sheath separates the hair shaft from the ORS (8).

There are three stages to the hair cycle. The active phase, which is also called the 


\section{VOLUME: 1 1, JANUARY-JUNE 2021}

anagen phase, is the first. The hair grows and thickens throughout this stage, replaces old hair, and continues to grow over time. The following is the second phase, the catagen, which is a transition period. It transforms active hair into dormant hair. The second stage lasts for up to three weeks. The third type of hair is telogen hair. Hair will remain in the scalp without growing and can be pulled out during this time. In addition, the hair grows during this time and can persist until new hair grows in and pulls the old hair out to fall out on its own $(7,8,9)$.

\section{ETIOLOGY OF HAIR LOSS}

\section{1) Hair loss induced by Hormonal} Changes

Female pattern hair loss is a prevalent disease that affects many women all over the world, but there isn't much research on it. The study of androgens has dominated hair biology to this point. Still, there is growing scientific and clinical evidence that non-androgen signals can impact the folliculosebaceous unit, particularly in women. Prior conceptions of estrogen activity and signaling in hair biology have been revised and modified since the identification of estrogen receptor beta. Estrogens are thought to influence hair development by influencing various other hormones. Some other factors which include cytokines malfunction and transcription factors are also responsible for changes in hair reproduction ultimately leading to hair loss (10).

\section{2) Hair loss induced by Nutritional}

\section{Changes}

Malnutrition and dietary deficits are common in people with inflammatory bowel disease. Symptoms such as poor absorption and poor digestion secondary to gastrointestinal tract inflammation or gut resection and increased intestinal losses are reasons for not taking enough nutrients. Nutritional inadequacy has long been known as a cause of alopecia in the absence of other pathologies. Alopecia has been proven to be caused by deficits in iron, zinc, and riboflavin in animal studies. The majority of human data pertains to PEM, linked to finer, more brittle hair with no color that depilates more easily and leads to growing hair slower. Hair loss in humans can be caused by iron deficiency and zinc deficiency. Among dermatologists, hair loss is one condition that is taken as a common issue and incorporates a significant mental and passionate effect on patients. Micronutrients play an imperative part in ordinary hair follicle advancement and safe cell work. Insufficiency of vitamins and minerals may speak to an adjustable 
hazard calculation related to the

improvement, avoidance, and treatment of alopecia $(11,12)$.

\section{Hair loss induced by Autoimmunity changes}

Many causes are related to the development of hair loss conditions. Genetic susceptibility is also linked to HLA antigen-presenting molecules, neuropeptide expression changes, and changes in the hair follicle's immuneprivileged condition. Both CD4+ and $\mathrm{CD} 8+\mathrm{T}$ cells can be linked somehow in hair loss condition, which appears to be a TH1 autoimmune disease. Though the auto-antigen is unknown, it could be linked to melanocytes. Immunomodulatory drugs that are now being developed and tested in clinical trials, notably for the treatment of psoriasis, as well as chemicals that modify the peripheral nervous system, should be investigated in the future (13).

\section{Hair loss induced by Inflammation}

Disorder related to hair loss can have a profound impact on our personality. Some of the psychological impacts of hair loss and baldness can be distressing and have a negative impact on a woman's personality and life quality. Many variables, including genetics and nutrition, contribute to hair loss. Inflammation in the body can be one
Inflammation develops when the body has to fight off invading microbes in the normal course. The body's immune system will mistake cells and organs for foreign creatures and attack them in rare situations. The body's immune system interprets hair follicles as intrusive components and destroys them frequently. Alopecia, or hair loss caused by inflammation, is the result of this. The immune system will harm the scalp at this time, and the hair roots will be unable to absorb proper nutrition. Inflammation and hair loss are linked in this way. A burning feeling and some red itchy areas are common symptoms of a damaged scalp caused by inflammation. In addition, peeling and other skin problems may occur on the scalp $(14,15)$.

\section{Hair loss induced by Metals and poisons}

The writing connecting the overdose of certain poisons to hair loss has been intensely centered on heavy metals. Within the final ten years, the writing still discusses heavy metals but presently also includes several non-metal poisons related to alopecia. Acknowledgment of harmful operators as a conceivable reason of alopecia, mindfulness of onset, and characteristic designs of hair loss condition or disorder will help the examiner or doctor, a better understanding of the 


\section{VOLUME: 1 1, JANUARY-JUNE 2021}

diagnosis of the condition. Despite many

studies, the etiology of essential unremitting telogen effluvium remains not completely caught on. Basic overwhelming metals are related to using impacts in people and other living beings. Be that as it may, they may lead to poisonous impacts when the introduction surpasses the higher passable limits. Therefore, People needed to survey the overwhelming metal and follow component levels in patients with persistent hair loss. Metals have a thickness at slightest five times more noteworthy than that of water with tall nuclear numbers. Be that as it may, they lead to poisonous impacts when the presentation surpasses the higher passable limits $(16,17)$.

\section{Hair Cycle Disruption}

The part and work related to the hair follicle are modern. The hair development cycle is disturbed when the hair follicles are being attacked by pathogens and harm the immune system. Many factors can be problematic and disrupt the hair cycle. Stress is one of them. Persistent stretch is related to hair development hindrance, expanded granulation of pole cells, and inflammation. Stress-causing substances are proved to be the major cause of developing issues in the hair development cycle in some studies. $(17,18)$.

\section{Genetic and Age-Related Hair Loss}

Alopecia in females and male thinning up top share a last common pathway of follicular relapse, but the latest information recommends the etiology isn't essentially the same in both genders. Androgens are meant to be responsible for male thinning up top and are also included within the etiology of design hair loss in a few ladies. In any case, other non-androgenic variables, not identified, play a part in causing female alopecia. Current studies suggest that many genetic and hereditary reasons are also involved in hair loss in both genders. Age is also one of the factors responsible for hair loss in both genders (18). As we get mature, one sign of maturity includes a change in the color of hair. Melanin is the pigment present in skin and hair which is responsible for producing pigment. Hair is developed by hair follicles that are present in the skin. If melanin is produced in low quantity, then it makes the hair reduce its color and the hair becomes gray. Melanin is supposed to be produced in less quantity during the process of maturity. Graying regularly starts within the 30s. Scalp hair regularly begins reducing the hair color at the sanctuaries and amplifies to the best of the scalp. Hair color gets to be lighter. Body and facial hair too turn Gray, but this frequently happens after scalp hair. 


\section{VOLUME: 1 1, JANUARY-JUNE 2021}

Graying is, to a great extent, decided by your qualities. In white people, there is already less melanin produced at a young age so in maturity their hair becomes gray before the other skin-toned people. Dietary supplements and other items will not halt or diminish the rate of color changes in old age $(19,20)$.

\section{Self-Inflicted Hair Loss}

Either deliberately or unknowingly, selfinflicted harm to hairs may cause hair misfortune in a few people. There are two sorts of self-induced hair loss (21-23):

Trichotillomania: This hair loss is commonly seen in childhood, more regularly in young girls than in boys. The individuals who compulsively and persistently drag or cull their hairs involvement trichotillomania. This suggests that this clutter encompasses a solid mental basis.

Traction Alopecia: This sort of hair misfortune is caused by the hair's nonstop and intemperate pulling and pressure due to a few sorts of hairstyling or hair frameworks settling on the hair itself.

\section{CONCLUSION}

Various reasons can cause alopecia or hair loss. The reasons include nutritional changes insufficient take of minerals and vitamins. Alopecia can be caused due to auto-immunity changes also. Genetic changes, toxicity, hormonal changes, and inflammation in the body are also the reasons for hair loss in men and women. Hair is a fundamental distinguishing feature of mammals as distinct species. Hair loss is caused when the immune system targets the hair follicles. Some external factors are also included in causing hair loss in people. Physiological and mental significance is linked to hair loss conditions. It secures against the bright beams of natural light and is considered to be served as a natural signaling work. Around the globe, women like to have their hair long and it is considered as a beautiful mark on their personality. Men like to have their hair thick and of good texture. It is in this way, barely shocking, that individuals with intemperate hair misfortune frequently look for therapeutic offer assistance. Hair loss can have a significant effect on our personality to be great. A few of the mental impacts of hair misfortune and hairlessness can be troubling and have a bad effect on a woman's quality of personality and life. 


\section{VOLUME: 11 , JANUARY-JUNE 2021}

\section{REFERENCES}

[1]Wolff H, Fischer TW, Blume-Peytavi U. The diagnosis and treatment of hair and scalp diseases. Deutsches Ärzteblatt International. 2016 May;113(21):377.

[2]Springer K, Brown M, Stulberg DL. Common hair loss disorders. American family physician. 2003 Jul 1;68(1):93-102.

[3] Sharma MK, Gupta S, Kumar R, Singhal AK, Jain SK, Sharma M. A clinicoepidemiological study of scalp hair loss in children (0-18 years) in Kota Region, SouthEast Rajasthan. Indian journal of dermatology. $2019 \mathrm{Jul} ; 64(4): 285$.

[4]Kıvrak Y, Yağcı İ, Üstündağ MF, Özcan H. Diffuse hair loss induced by sertraline use. Case reports in psychiatry. 2015 Sep 17;2015.

[5]Phillips TG, Slomiany WP, Allison R. Hair loss: common causes and treatment. American family physician. 2017 Sep 15;96(6):371-8.

[6]Al-Aradi, I. and Al-Ghareeb, M., Hair Fall: Common Causes and Treatment Modalities.

[7]Messenger AG. The control of hair growth: an overview. Journal of Investigative Dermatology. 1993 Jul 1;101(1): S4-9.

[8]Percie du Sert N, Hurst V, Ahluwalia A, Alam S, Avey MT, Baker M, Browne WJ, Clark A, Cuthill IC, Dirnagl U, Emerson M. The ARRIVE guidelines 2.0: Updated guidelines for reporting animal research. Journal of Cerebral Blood Flow \& Metabolism. 2020 Sep;40(9):1769-77.

[9]Buffoli B, Rinaldi F, Labanca M, Sorbellini E, Trink A, Guanziroli E, Rezzani R, Rodella LF. The human hair: from anatomy to physiology. International journal of dermatology. 2014 Mar;53(3):331-41.

[10] Lin RL, Garibyan L, Kimball AB, Drake LA. Systemic causes of hair loss. Annals of medicine. 2016 Aug 17;48(6):393-402.

[11] Rushton DH. Nutritional factors and hair loss. Clinical and Experimental Dermatology: Clinical dermatology. 2002 Jul;27(5):396-404.

[12] Goldberg LJ, Lenzy Y. Nutrition and hair. Clinics in dermatology. 2010 Jul $1 ; 28(4): 412-9$.

[13] Hordinsky M, Ericson M. Autoimmunity: alopecia areata. InJournal of Investigative Dermatology Symposium Proceedings 2004 Jan 1 (Vol. 9, No. 1, pp. 73-78). Elsevier. 


\section{VOLUME: 1 1, JANUARY-JUNE 2021}

[14] Peyravian N, Deo S, Daunert S, Jimenez JJ. The inflammatory aspect of male and female pattern hair loss. Journal of inflammation research. 2020;13:879.

[15] Valdebran M, Mo J, Elston DM, Doan L. Pattern hair loss: assessment of inflammation and fibrosis on histologic sections. Journal of the American Academy of Dermatology. 2020 Mar 1;82(3):757-8.

[16] Trüeb RM. Systematic approach to hair loss in women. JDDG: Journal der Deutschen Dermatologischen Gesellschaft. 2010 Apr;8(4):284-97.

[17] Trüeb RM. Diffuse hair loss. InHair growth and disorders 2008 (pp. 259-272). Springer, Berlin, Heidelberg.

[18] Cotsarelis G, Millar SE. Towards a molecular understanding of hair loss and its treatment. Trends in molecular medicine. $2001 \mathrm{Jul}$ 1;7(7):293-301.

[19] Whiting DA. Male pattern hair loss: current understanding. International journal of dermatology. 1998 Aug 1;37(8):561-6.

[20] Westgate GE, Botchkareva NV, Tobin DJ. The biology of hair diversity. International journal of cosmetic science. 2013 Aug;35(4):329-36.

[21] Harth W, Blume-Peytavi U. Psychotrichology: psychosomatic aspects of hair diseases. JDDG: Journal der Deutschen Dermatologischen Gesellschaft. 2013 Feb;11(2):125-35.

[22] Júnior AC, Katzer T, Ramos DG. Three cases of hair loss analyzed by the point of view of the analytical psychology. International journal of trichology. 2017 Oct;9(4):177.

[23] Gupta MA, Gupta AK, Haberman HF. The self-inflicted dermatoses: a critical review. General Hospital Psychiatry. 1987 Jan 1;9(1):45-52. 